\title{
Evaluation of children's dental anxiety levels at a kindergarten and at a dental clinic
}

\section{Gulser KILINÇ(a) \\ Aynur AKAY(b) \\ Ece EDEN(c) \\ Nilgün SEVINÇ(d) \\ Hülya ELLIDOKUZ(e)}

(a) Dokuz Eylul University, Medical Faculty, Pediatric Dentistry Inciaralti, Izmir, Turkey.

(b)Dokuz Eylul University, School of Medicine, Department of Pediatric and Adolescent Psychiatry Inciralti, Izmir, Turkey.

(c)Ege University, Faculty of Dentistry, Department of Pediatric Dentistry Clinic, Izmir, Turkey.

(d) Dokuz Eylul University, School of Medicine, Pediatric Clinic Inciaralti, Izmir, Turkey.

(e)Dokuz Eylul University, School of Medicine, Department of Biostatistics and Medical Informatics Inciaralti, Izmir, Turkey.

Declaration of Interests: The authors certify that they have no commercial or associative interest that represents a conflict of interest in connection with the manuscript.

\section{Corresponding Author:}

Gulser Kilinc

E-mail: gulser.kilinc@deu.edu.tr

DOI: 10.1590/1807-3107BOR-2016.vol30.0072

Submitted: Aug 25, 2015

Accepted for publication: Jan 19, 2016

Last revision: Apr 28, 2016
Abstract: This study evaluated the dental anxiety levels of preschool children at a kindergarten and at a dental clinic. The anxiety levels of ninety 4-6-year-old $(4.99 \pm 0.81)$ preschool children were evaluated according to pulse rates, the facial image scale (FIS), the Venham picture test (VPT), and the Frankl behavior rating scale. The children's mothers were asked to complete the state-trait anxiety inventory (STAI) forms 1 and 2 (STAI 2 and STAI 2). The sample t-test, Mann-Whitney U test, and Pearson's correlation test were used. A statistically significant difference was observed between the children's pulse rates when measured at the dental clinic and those when measured at the kindergarten $(p<0.001)$. Although the results were not statistically significant, more negative facial expressions were observed in the children at the dental clinic than in those at the kindergarten when assessed using FIS and VPT ( $p=0.090$ and $p=0.108$, respectively). There was a statistically significant correlation between the transient anxiety levels (STAI 1) of mothers and the VPT scores of their children evaluated at the dental clinic $(r=0.506, p<0.001)$. The continuous anxiety level of the mothers of males was found to be significantly higher $(p=0.033)$ than that of the mothers of females (STAI 2). Although the children had been informed about dentistry and were introduced to a dentist at the kindergarten, their anxiety levels seemingly increased as they arrived at the dental clinic. The significant increase observed in the children's pulse rates was a physical indicator that their anxiety levels had increased. It can be concluded that the children felt more anxious at the dental clinic that at the kindergarten.

Keywords: Dental Anxiety; Child, Preschool; Fear; Behavior; Parents.

\section{Introduction}

Patient anxiety can be a major problem in all branches of medicine, including dentistry. It can present problems for both the dentist and patients, particularly in pediatric dentistry. Factors specific to dentistry that can negatively affect a child on an emotional level include dental instruments with which the child has no prior experience, treatment methods, pain arising from treatments, fear caused by these treatments, and unfamiliar adults working as staff at the dental clinic. In addition, negative experiences that a child may have had at an early age and his/her interactions with the environment are factors that increase anxiety. ${ }^{1,2,3}$ 
Adverse childhood dental treatment experiences often reduce the frequency of pediatric patients' visits to the dentist in the future, which can subsequently lead to poor oral hygiene. ${ }^{3}$

Dental anxiety is a complex condition and has various components. As previously implied, many factors in addition to past traumatic dental experiences, including personality, gender, age, and education level, may affect patients' dental anxiety levels. ${ }^{4,5}$ Some studies have shown that dental anxiety affects daily life and creates problems in family relationships and social activities. ${ }^{6,7}$

The dentist's awareness of the patient's anxiety level prior to treatment prepares him/her for the patient's reactions and allows him/her to take precautions to reduce the patient's anxiety level. This is crucial, particularly when dealing with pediatric patients. To help children cope with anxiety, the dentist should know more about the etiology, level of dental anxiety, and psychology of the child. Studies on dental anxiety have indicated that etiology involves multiple factors. ${ }^{8,9}$

The early identification of dental anxiety in a patient is important for dentists so that they can properly approach their patients. ${ }^{8}$ For this reason, many methods have been developed to assess dental anxiety. Some physiological changes may be an indicator of anxiety, such as an increase in pulse rate or salivary cortisol levels. ${ }^{10}$ It is easy to measure the pulse of a child in any setting, which gives this method an advantage. The most common scales used in children under 6 years of age are projective measures, i.e., the children are shown some pictures and are then asked to describe what they feel. . $2,3,11^{2}$

Many studies have used facial images to assess the feelings of pediatric patients. ${ }^{3,6}$ The most important step when using such a scale is to validate it with another scale that has been previously validated for the group under study. ${ }^{2,3,6}$ In the case of very young children, the Venham picture test (VPT) is often used to avoid the need for extensive oral communication with children. VPT uses eight cards, each of which shows two pictures portraying opposite feelings. $3,6,11$ Dentists also often use the Frankl behavior rating scale, which evaluates children's behavior based on observation. More specifically, according to this behavioral scoring method, the child is observed and assigned a score. ${ }^{12,13}$ The facial image scale (FIS) is also commonly used by dentists. ${ }^{2,6,12,14}$ It comprises a row of five faces ranging between "very unhappy" and "very happy" and numbered from 5 to 1, and it aims to assess the state of anxiety. Each child is asked to point to the face that they feel most closely reflects their feelings at that moment.

The most effective and reliable method for truly assessing dental anxiety levels involves the parental interpretation of information obtained from pediatric patients. However, this information will be biased by the parents' feelings about past experiences, the anxiety caused by past and current experiences, and the expectations from the current process..$^{15}$ In fact, dental anxiety in preschool children can be affected by the dental anxiety experienced by their mothers as well as by general anxiety. ${ }^{16}$ Corkey and Freeman ${ }^{17}$ demonstrated that the relationship between a mother and her child in a group of 6-year-old pediatric patients affects their psychological development, compliant behavior at a dental clinic, and oral hygiene.

This study was designed to assess the dental anxiety levels of children at a kindergarten and at a dental clinic to determine the effects of familial factors, gender, and age on their dental anxiety levels.

\section{Methodology}

The study population comprised 90 children between the ages of 4 and 6 years enrolled at Dokuz Eylul University Kindergarten. Every child enrolled at the kindergarten and his/her respective family members were included. The study was approved by the ethics committee (ref number 156-IOÇ/2010), and written consents were obtained from the parents. FIS, VPT, and pulse rate measurements were performed both at the kindergarten classroom and at the dental clinic, whereas the Frankl Behavior Rating Scale was only applied at the dental clinic.

\section{At the kindergarten}

A dentist and physician visited the kindergarten classroom between 10:30 and 11:00 AM and introduced themselves before inviting each child for an interview in a room next to the classroom. The children's initial anxiety levels were evaluated by the dentist using FIS and VPT, and pulse rate measurements 
were performed by the physician; the children were individually examined while they remained seated to establish a baseline pulse rate. After the initial screening, the dentist informed the children and teachers about mouth and teeth care and tooth brushing methods via a story.

\section{At the dental clinic}

The children visited the dental clinic in groups of 10 and were supervised by their teachers. During this visit, the children were introduced to operations at the dental clinic and were informed about dental examinations and the application of topical fluoride varnish. In addition, each child was given an oral examination to determine their current dental state (e.g., cavities, missing teeth, number of dental fillings, and oral hygiene). Five percent sodium fluoride varnish was applied on all their teeth. A pediatrician measured the children's pulses while they were sitting on a dental chair, and the dentist assessed their anxiety levels using FIS and VPT after the oral examination and fluoride application. The Frankl behavior rating scale was used by the dentist after the oral examination and fluoride application.

\section{At home}

The mothers of the children filled a questionnaire, which included questions about the child and the mother's sociodemographic characteristics as well as question about their children's tooth brushing frequency. The state-trait anxiety inventory [STAI form 1 (STAI 1) and form 2 (STAI 2)] for the mothers were filled at home to assess their anxiety levels. In total, 66 mothers completed the STAI 1 and 2. These forms help measure the children's transient and continuous anxiety levels. Although 24 mothers stated that they did not want to complete these forms, all mothers signed the informed consent document allowing their children to participate.

\section{Scales used}

FIS comprises five pictures showing different emotions. The unhappiest face was assigned a score of 5 and the happiest face was assigned a score of $1.2,6$

VPT includes the use of eight different cards. Each card contains two pictures, i.e., a child who is anxious and another who is not. In this test, each picture showing an anxious child was assigned a score of 1 and the opposite was assigned a score of 0 .

For both tests (FIS and VPT), the children were asked to choose the picture best illustrating what they felt at that moment. The total score for VPT was recorded. $3,6,1,18$

The Frankl behavior rating scale is a behavioral scoring method based on visual evaluation by the dentist. The child's behavior is categorized into one of the following four groups: strictly negative, negative, positive, or strictly positive. ${ }^{11,12,13}$

STAI 1 and 2 measure the children's transient and continuous anxiety levels. This inventory is easy to apply because the child can answer the questions himself/herself. Positive and negative items are scored from 1 to 4 , with 1 referring to "not at all" and 4 referring to "too much". 19

As previously indicated, each child's pulse rate was measured while they remained in a sitting position. A pediatrician performed the measurement by counting the heart rate from the radial artery for $1 \mathrm{~min}$ after the child had rested for at least $15 \mathrm{~min}$.

\section{Statistical analysis}

Statistical package for the social sciences (SPSS) 15.0 manufactured by IBM (Chicago, USA) was used for the statistical analysis. Continuous variables (pulse rate, VPT, FIS, and STAI) were compared using the independent sample t-test. The comparison of categorical variables (Frankl behavior rating) was done by the Mann-Whitney U test. The paired sample t-test was used for pulse variables (baseline and clinical results). In addition, the Pearson's correlation test was used to determine the correlation between the anxiety scale scores. The continuous variables are expressed as mean \pm standard deviation, whereas the categorical variables are expressed in terms of percentage. In all analyses, $p<0.05$ was accepted as statistically significant.

\section{Results}

The average age of the included children was $4.99 \pm 0.81$ years (between 4 and 6 years). In terms of gender distribution, $51 \%$ of the children were males and $49 \%$ were females. Sociodemographic characteristics 
revealed that $51.1 \%$ of the children were the only child in their family. Most mothers were university graduates $(77.8 \%)$, and they mostly $(90 \%)$ reported no history of dental anxiety within the family; further, $73.3 \%$ of the children never visited the dentist.

Table 1 shows the FIS and VPT scores and the pulse rates at the kindergarten and at the dental clinic, as well as the Frankl behavior rating scale scores at the dental clinic according to gender. No statistically significant differences were observed in the gender-based analysis.

Table 2 shows the pulse rates and FIS and VPT scores of the children at the kindergarten and at the dental clinic. A statistically significant difference was observed among the children's pulse rates when measured at the kindergarten and those when measured at the dental clinic $(p<0.001)$. More specifically, the children's average pulse rate in the dental clinic environment was higher than that in the kindergarten environment. Evaluations based on FIS revealed that the children exhibited more negative facial expressions at the dental clinic than at the kindergarten, but this difference was not statistically significant $(p=0.090)$. Similarly, the VPT scores were more negative at the dental clinic, but there was no statistically significant difference $(p=0.108)$. Nevertheless, a statistically significant positive correlation was observed between the FIS scores and VPT scores at the kindergarten $(r=0.268$, $p=0.011)$, and this was even more evident at the dental clinic $(r=0.502, p<0.001)$.

Table 3 shows the children's acceptance of dental treatment according to the Frankl behavior rating scale. Three children refused to sit on the dental chair, and two more children refused fluoride application after the oral examination.

STAI 1 and 2, which help measure the children's transient and continuous anxiety levels, respectively, were completed by 66 mothers; as previously noted, 24 mothers stated that they did not want to complete these forms. The relevant data are summarized in Table 4. Interestingly, the continuous anxiety levels of the mothers of males was found to be significantly higher $(p=0.033)$ than those of the mothers of females (STAI 2). The children's anxiety status and its relation to their mothers' anxiety levels revealed that there was a statistically significant correlation between the transient anxiety levels (STAI 1) of mothers and VPT scores of their children evaluated at the dental clinic $(\mathrm{r}=0.506, \mathrm{p}<0.001)$.

Table 1. The pulse rates, facial images scale (FIS) scores, Venham picture test (VPT) scores scores, and Frankl behavior rating scale scores according to gender at the kindergarten before treatment and at the dental clinic after treatment.

\begin{tabular}{|c|c|c|c|c|c|c|c|c|}
\hline \multirow{3}{*}{ Gender } & \multirow{3}{*}{$\mathrm{n}$} & \multicolumn{3}{|c|}{ Pretreatment } & \multicolumn{4}{|c|}{ After the Treatment } \\
\hline & & \multicolumn{3}{|c|}{ The kindergarten environment } & \multicolumn{4}{|c|}{ The clinical environment } \\
\hline & & Pulse & FIS & VPT & Pulse & FIS & VPT & $\begin{array}{l}\text { Frankl behavior } \\
\text { rating scale score }\end{array}$ \\
\hline Female & 44 & $95.18 \pm 12.9$ & $2.93 \pm 0.7$ & $2.89 \pm 0.7$ & $107.00 \pm 12.9$ & $3.36 \pm 0.6$ & $3.09 \pm 0.6$ & $4.63 \pm 1.75$ \\
\hline Male & 46 & $95.20 \pm 11.0$ & $2.83 \pm 0.8$ & $2.83 \pm 0.8$ & $109.24 \pm 11.7$ & $3.13 \pm 0.5$ & $3.23 \pm 0.6$ & $4.19 \pm 1.55$ \\
\hline$p^{*}$ & - & 0.996 & 0.529 & 0.719 & 0.393 & 0.070 & 0.297 & 0.210 \\
\hline
\end{tabular}

*Statistically significant $(p<0.05)$.

Table 2. The entire sample population's pulse rates, facial images scale (FIS) scores, Venham picture test (VPT) scores at the kindergarten before treatment and at the clinic after treatment.

\begin{tabular}{lcccc}
\hline Variable & $\mathrm{n}$ & Pulse mean \pm SD & FIS mean \pm SD & VPT mean \pm SD \\
\hline At the kindergarten & 90 & $95.19 \pm 11.9$ & $2.88 \pm 0.7$ & $2.86 \pm 0.6$ \\
At the vlinic & 90 & $108.14 \pm 12.3$ & $3.24 \pm 0.6$ & $3.16 \pm 0.7$ \\
$\mathrm{p}^{*}$ & - & $\mathrm{p}<0.001^{*}$ & $\mathrm{p}=0.090$ & $\mathrm{p}=0.108$ \\
\hline
\end{tabular}

SD: Standard deviation; *Statistically significant $(p<0.05)$. 
Table 3. Frankl Behavior Rating Scale scores for children according to their acceptance of oral examination and fluoride application.

\begin{tabular}{lcc}
\hline \multirow{2}{*}{ Variable } & Acceptance of oral examination & Acceptance of fluoride application \\
\cline { 2 - 3 } & $\mathrm{n}(\%)$ & $\mathrm{n}(\%)$ \\
\hline Definitely negative & $3(3.3)$ & $5(5.6)$ \\
Negative & $5(5.6)$ & $9(10.0)$ \\
Positive & $74(82.2)$ & $71(78.8)$ \\
Definitely positive & $8(8.9)$ & $5(5.6)$ \\
Total & $90(100)$ & $90(100)$ \\
\hline
\end{tabular}

Table 4. Scores on the state-trait anxiety inventory (STAI) 1 (momentary) and STAI 2 (continuous) tests according to gender of the children.

\begin{tabular}{lcccc}
\hline & Gender & $n$ & Mean \pm SD & $p^{*}$ \\
STAI 2 (continuous) & Male & 35 & $48.97 \pm 9.69$ & $0.033^{*}$ \\
\multirow{2}{*}{ STAI 1 (momentary) } & Female & 31 & $44.32 \pm 7.61$ & 0.090 \\
& Male & 35 & $31.88 \pm 12.88$ & 0.225 \\
\hline
\end{tabular}

SD: Standard deviation; *Statistically significant $(p<0.05)$.

\section{Discussion}

Many studies have evaluated dental anxiety levels in children. ${ }^{6,11,12,14,16,17}$ However, it is difficult to measure the anxiety levels of children. In an effort to more accurately assess anxiety levels in children, dentists typically utilize scales, which are particularly designed for use with children. These scales can quickly provide feedback on children's anxiety levels prior to treatment. Nevertheless, the dental anxiety level is difficult to measure because it is a subjective issue that differs among individuals. For this reason, in the present study, four different scales that have been recognized worldwide were used. $2,6,11,13,20$

Similar to the present study, many studies have used facial images to assess feelings in pediatric patient groups. ${ }^{2,3,6}$ The child is shown some pictures and is then asked to choose the picture that best reflects his/her feelings at that moment. The verbal skills of children in this age group are often quite limited; therefore, we used FIS and VPT, which are based on facial images and have proven to be valid instruments in many studies. ${ }^{23,6,11}$

The most important consideration when using a new scale is the comparison of results from its use with those from another scale that has been validated in previous studies. ${ }^{3,6,21}$ Therefore, we paired the measurement of pulse rates with four different well-recognized scales to evaluate the pulse rate as a concrete indicator of anxiety. This study has shown that the children's anxiety levels, as measured by the pulse rate as well as four other scales, increased at the dental clinic, even though they had already met the dentist and received information at the kindergarten.

When the results of this study were examined in terms of gender, no statistically significant differences were observed regarding pulse rates, FIS scores, VPT scores, or Frankl behavior rating scores. Similar to our findings, Buchanan and Niven ${ }^{6}$ found that there were no significant differences in terms of gender regarding the scores that measure dental anxiety levels with FIS and VPT. However, another study found that female adolescents experience more dental anxiety levels than their male counterparts. ${ }^{7}$ In addition, one study showed that males experience higher dental anxiety levels in preschool groups, whereas females experience higher dental anxiety levels in school-age groups. ${ }^{22}$

A statistically significant positive correlation was observed between the FIS and VPT scores in the present study. More specifically, as the FIS scores at the dental clinic became more negative, the VPT scores also became more negative. A strong correlation between the FIS and VPT scores was also observed in the study by Buchanan and Niven ${ }^{6}$ Our 
study showed that $8.9 \%$ of the children received a "negative and definitely negative" Frankl behavior rating score. Yamada et al. ${ }^{16}$ reported $8 \%$ of the children receiving this score, whereas Nakai et al. ${ }^{23}$ reported $15 \%$. In our study, $8.9 \%$ of the children refused the oral examination and $15.6 \%$ rejected fluoride application, which is in agreement with the above articles.

Studies have shown that the pulse rate rises in clinical settings. ${ }^{10,24}$ Sowjanya et al. ${ }^{24}$ and Guinot Jimeno et al. ${ }^{25}$ reported that the highest pulse rate is observed during local anesthesia administration and extractions. In the present study, no treatments associated with pain (e.g., shots, fillings, or extractions) were administered to the children at the dental clinic. Within the population in our study, $73.3 \%$ of the children had no prior dental experience. Although the children only underwent oral examinations and topical fluoride varnish applications, we observed a significant increase in their pulse rates, which allowed us to physically observe the increase in their anxiety levels. These pulse rate increases, which indicate increases in adrenergic activity caused by anxiety, showed us that the children's anxiety levels were higher in the clinical environment than in the kindergarten environment. In spite of information on oral and dental health that they were given, the pulse rates of the children in the study group were higher at the dental clinic.

Although the results from the FIS measurement were not statistically significant, it was noted that the children exhibited more negative facial expressions at the dental clinic than at the kindergarten, corresponding to the observed increase in their pulse rates. This study has also shown that education on oral health care for children at younger ages in the kindergarten environment is not enough for reducing dental anxiety levels. In fact, further education and comfort are crucial to alleviate children's fears in the clinical environment.

This study also found a statistically significant correlation between mothers' anxiety levels (as measured by the momentary STAI 1 and the children's VPT scores at the dental clinic $(\mathrm{p}<0.001)$. The structured review and meta-analysis revealed that there was a significant relationship between parental and child dental fear, particularly in children who are 8 years and younger. ${ }^{14}$ In contrast, Leal et al. ${ }^{26}$ reported that there was no correlation between parents' anxiety levels and those of their children aged between 4 and 12 years.

Many studies have established a strong relationship between age and dental anxiety levels. ${ }^{1,2,3,6}$ In fact, these studies reported that children in young age groups are typically more anxious than older children, which is consistent with our findings. ${ }^{16,24}$ The relationship between age and dental anxiety levels is inversely proportional.

Patients' age and gender and family's educational status and socioeconomic level are factors that can affect dental anxiety levels. ${ }^{22,24,27,28,29}$ In this study, most mothers were highly educated, and there was no reported history of phobic tendencies with dental treatment, so we cannot make any claims regarding a relationship between a child's dental anxiety level and their family's sociodemographic characteristics.

Every attempt should be taken to relieve children's anxiety and to comfort them while at the dental clinic. The dentist's knowledge about the anxiety levels of a child and his/her mother before treatment will help the dentist prepare for reactions that can occur with any given anxiety level and will allow the dentist to take precautions to reduce a child's anxiety level if necessary.

\section{Conclusion}

According to our findings, children were more anxious at the dental clinic than at the kindergarten. Therefore, children should be allowed to express their concerns during their routine visits to the dentist and during their dental treatment. We also recommend approaching children in a comforting manner.

\section{Acknowledgments}

This research was first presented as a poster at the 25th National Congress of Child and Adolescent Psychiatry. Dr. Akay is Advisory Board Member and has served on speakers' bureaus for Janssen and Lilly Pharmaceutical Companies. The other authors declare that they have no competing financial interests. This work was not supported by any grant. 


\section{References}

1. Humphris GM, Milsom K, Tickle M, Holbrook $\mathrm{H}$, Blinkhorn A. A new dental anxiety scale for 5-year-old children (DA\%) description and concurrent validity. Health Educ J. 2002;61(1):5-19. doi:10.1177/001789690206100102

2. Kuşcu OO, Akyüz S. Children's preferences concerning the physical appearance of dental injectors. J Dent Child (Chic). 2006;73(2):116-21.

3. Ramos-Jorge ML, Marques LS, Pavia SM, Serra-Negra JM, Pordeus IA. Predictive factors for child behaviour in the dental environment. Eur Arch Paediatr Dent. 2006;7(4):253-7. doi:10.1007/BF03262561

4. Thomson WM, Locker D, Poulton R. Incidence of dental anxiety in young adults in relation to dental treatment experience. Community Dent Oral Epidemiol. 2000;28(4):289-94. doi:10.1034/j.1600-0528.2000.280407.x

5. Locker D, Poulton R, Thompson WM. Psychological disorders and dental anxiety in a young adult population. Community Dent Oral Epidemiol. 2001;29(6):456-63. doi:10.1034/j.1600-0528.2001.290607.x

6. Buchanan H, Niven N. Validation of a Facial Image Scale to assess child dental anxiety. Int J Paediatr Dent. 2002;12(1):47-52. doi:10.1046/j.0960-7439.2001.00322.x

7. Brukiene V, Aleksejuniene J. Balciuniene I. Is dental treatment experience related to dental anxiety? A croos-sectional study in Lithuanian adolescents. Stomatologija. 2006;8(4):108-15.

8. Cardoso CL, Loureiro SR, Nelson-Filho P. Pediatric dental treatment: manifestations of stress in patients, mothers and dental school students. Braz Oral Res. 2004;18(2):150-5. doi:10.1590/S1806-83242004000200011

9. Carrillo-Díaz M, Crego A, Armfield JM, Romero M. Self-assessed oral health, cognitive vulnerability and dental anxiety in children: testing a mediational model. Community Dent Oral Epidemiol. 2012;40(1):8-16. doi:10.1111/j.1600-0528.2011.00646.x

10. Schriks MC, Amerongen WE. Atraumatic perspectives of ART: psychological and physiological aspects of treatment with and without rotary instruments. Community Dent Oral Epidemiol. 2003;31(1):15-20. doi:10.1034/j.1600-0528.2003.00021.x

11. Afshar H, Baradaran Nakhjavani Y, Mahmoudi-Gharaei J, Paryab M, Zadhoosh S. The effect of parental presence on the 5 year-old children's anxiety and cooperative behavior in the first and second dental visit. Iran J Pediatr. 2011;21(2):193-200.

12. Shinohara S, Nomura Y, Shingyouchi K, Takase A, Ide M, Moriyasu $\mathrm{K}$ et al. Structural relationship of child behavior and its evaluation during dental treatment. J Oral Sci. 2005;47(2):91-6. doi:10.2334/josnusd.47.91

13. Sharath A, Rekka P, Muthu MS, Rathna Prabhu V, Sivakumar N. Children's behavior pattern and behavior management techniques used in a structured postgraduate dental program. J Indian Soc Pedod Prev Dent. 2009;27(1):22-6. doi:10.4103/0970-4388.50812

14. De Menezes Abreu DM, Leal SC, Mulder J, Frencken JE. Dental anxiety in 6-7-year-old children treated in accordance with conventional restorative treatment, ART and ultra-conservative treatment protocols. Acta Odontol Scand. 2011;69(6):410-6. doi:10.3109/00016357.2011.572561

15. Themessl-Huber M, Freeman R, Humphris G, MacGillivray S, Terzi N.. Empirical evidence of the relationship between parental and child dental fear:a structured review and meta-analysis. Int J Paediatr Dent. 2010;20(2):83-101. doi:10.1111/j.1365-263X.2009.00998.x

16. Yamada MK, Tanabe Y, Sano T, Noda T. Cooperation during dental treatment: the Children's Fear Survey Schedule in Japanese children. Int J Paediatr Dent. 2002;12(6):404-9. doi:10.1046/j.1365-263X.2002.00399.x

17. Corkey B, Freeman R. Predictors of dental anxiety in six-year-old children: findings from a pilot study. ASDC J Dent Child. 1994;61(4):267-71.

18. Venham L, Bengston D, Cipes M. Children's response to sequential dental visits. J Dent Res. 1977;56(5):454-9. doi:10.1177/00220345770560050101

19. Spielberger CD, Gorsuch RL, Lushene RE. Manual for the state-trait anxiety inventory. Palo Alto: Consulting Psychologists Press; 1970.

20. Marteau TM, Bekker H. The development of a six-item short-form of the state scale of the Spielberger State-Trait Anxiety Inventory (STAI). Br J Clin Psychol. 1992;31(3):301-6. doi:10.1111/j.2044-8260.1992.tb0099

21. Topaloglu-Ak A, Eden E, Frencken JE. Perceived dental anxiety among schoolchildren treated through three caries removal approaches. J Appl Oral Sci. 2007;15(3):235-40. doi:10.1590/S1678-77572007000300015

22. Holst A, Crossner CG. Direct ratings of acceptance of dental treatment in Swedish children. Community Dent Oral Epidemiol. 1987;15(5):258-63. doi:10.1111/j.1600-0528.1987.tb00533.x

23. Nakai Y, Hirakawa T, Milgrom P, Coolidge T, Heima M, Mori $Y$ et al. The children's fear survey schedule- dental subscale in Japan. Community Dent Oral Epidemiol. 2005;33(3):196-204. doi:10.1111/j.1600-0528.2005.00211.x

24. Sowjanya V, Tandon S, Tharian E. Physiological response to dental anxiety in children. J Indian Soc Pedod Prev Dent. 1996;13(1):13-7.

25. Guinot Jimeno F, Mercadé Bellido M, Cuadros Fernández C, Lorente Rodríguez AI, Llopis Pérez J, Boj Quesada JR. Effect of audiovisual distraction on children's behavior anxiety and pain in the dental setting. Eur J Paediatr Dent. 2014;15(3):297-302. 
- Evaluation of children's dental anxiety levels at a kindergarten and at a dental clinic

26. Leal AM, Serra KG, Queiroz RCS, Araujo MAR, Maia Filho EM. Fear and/or anxiety of children and parents associated with the dental environment. Eur J Paediatr Dent. 2013;14(4):269-72.

27. Raadal M, Milgrom P, Weinstein P, Mancl L, Cauce AM. The prevalence of dental anxiety in children from low-income families and its relationship to personality traits. J Dent Res. 1995;74(8):143943. doi:10.1177/00220345950740080201
28. Folayan MO, Idehen EE, Ufomata D. The effect of sociodemographic factor on dental anxiety in children seen in a suburban Nigerian hospital. Int J Paediatr Dent. 2003;13(1):20-6. doi:10.1046/j.1365-263X.2003.00411.x

29. Armfield JM. The extent and nature of dental fear and phobia in Australia. Aust Dent J. 2010;55(4):368-77. doi:10.1111/j.1834-7819.2010.01256.x 